\title{
EXPERIMENTAL ANALYSIS OF STIFFNESS OF THE RIVETED STEEL RAILWAY BRIDGE DECK MEMBERS' JOINTS
}

\author{
Jozef GOCÁL ${ }^{1 *}$, Richard HLINKA ${ }^{1}$, Jozef JOŠT ${ }^{1}$, František BAHLEDA ${ }^{1}$ \\ ${ }^{1}$ Department of Structures and Bridges, Faculty of Civil Engineering, University of Žilina, Univerzitná \\ 8215/1, 01026 Žilina. \\ corresponding author: jozef.gocal@fstav.uniza.sk.
}

\section{Abstract}

The paper deals with the real behaviour of the riveted steel railway bridge deck members' connections with respect to their bending stiffness. Attention is paid to the stringer-to-cross beam connection as well as the cross beam-to-main girder connection. The stiffness of the two connections is investigated on the basis of evaluation of the experimentally determined stress response of the observed structural members to the actual traffic load on an existing railway bridge.
\end{abstract}

\section{Keywords:}

Riveted steel railway bridge; Stringer-to-cross beam connection;

Cross beam-to-main girder connection;

Experimental measurements; Joint stiffness.

\section{Introduction}

In the frame of preparation of the guidelines for determination of the loading capacity of the railway bridges, the many investigations of the stringer-to-cross-beam connections behaviour of the railway bridges with open bridge decks were carried up [1], [2], [3] in order to avoid approximation of those joints by means of complicated $2 \mathrm{D}$ modelling and to use simplified $1 \mathrm{D}$ model for global analyses of these types of bridges. Mutual connection between those deck members with I-shaped cross sections, which is generally realised only by connecting the webs, used to be modelled as nominally hinged in global analysis of the superstructure at the time of its designing. However, the actual behaviour of riveted joints is usually more complicated but not sufficiently known. The results of analysis presented in [3] showed very complicated behaviour of the observed joints according to the structural solutions of the stringer-to-cross-beam connections. In order to clarify the real behaviour of these joints, several experimental measurements on selected riveted steel railway bridges as well as on laboratory specimens have been realized, focused also on the fatigue behaviour of these joints. The fatigue behaviour of riveted joints of steel railway bridges has been the subject of research also abroad [4], [5], [6].

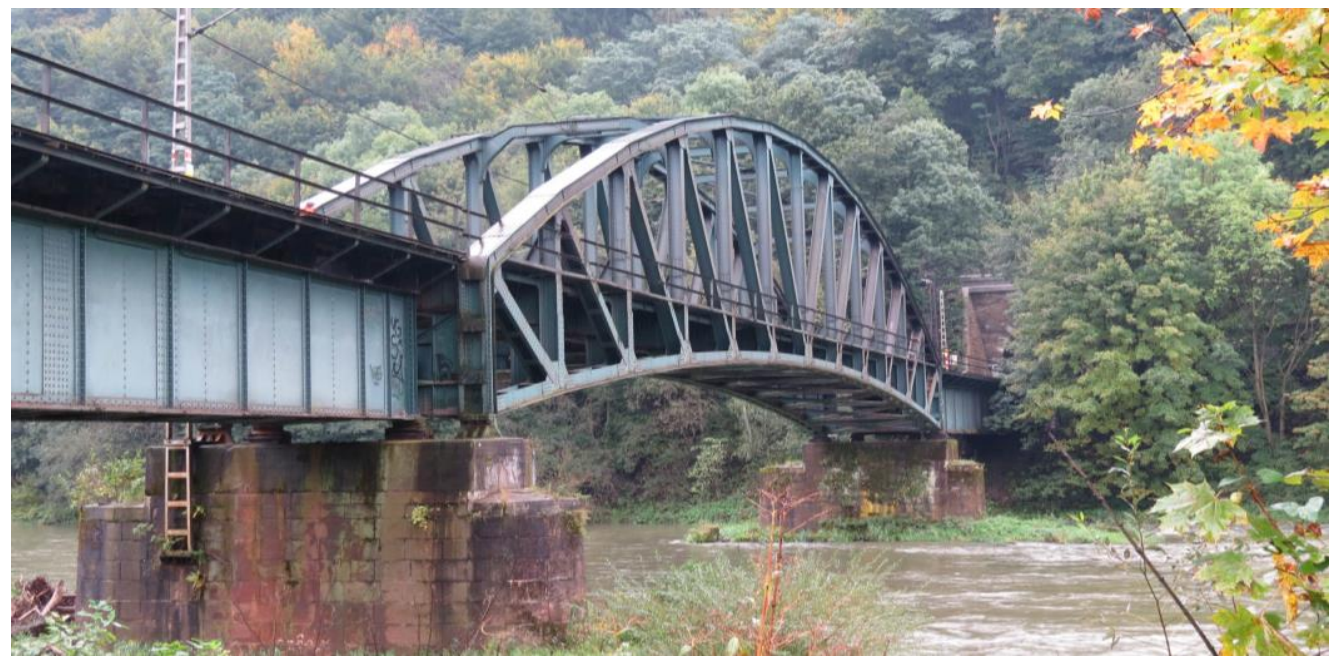

Fig. 1: View at the railway bridge over the Vah River near Strečno. 
The presented research is focused on the analysis of real stiffness of the stringer-to-crossbeam connection and the cross-beam-to-main-girder connection, as well. The aim is to suggest possible recommendations for practical numerical modelling of these types of bridges. In this paper, there are presented the results of experimental measurements executed on the steel truss bridge situated in km 324.386 of the track Košice - Žilina, near the Strečno tunnel (Fig. 1).

\section{Brief description of the bridge}

The bridge consists of three simply supported superstructures with theoretical spans $29.4+$ $57.4+29.4 \mathrm{~m}$ (Fig. 2). The edge spans are designed as plate girder bridges with upper open bridge deck, consisting of cross-beams and continuous stringers deployed on the cross-beams. Concerning the focus of the research, the bridge superstructure applied in the medium span is more interesting.

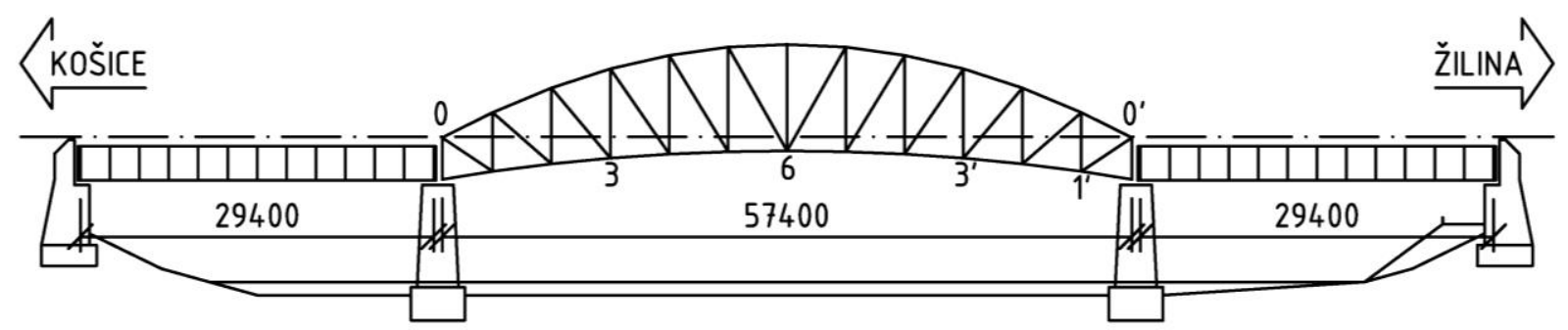

Fig. 2: Longitudinal disposition of the bridge.

It is designed as truss bridge with intermediate open bridge deck consisting of the cross-beams and stringers embedded between them (Fig. 3). It is situated in a transition curve to the arc with radius $108 \mathrm{~m}$, which has reflected in variable track axes distance from the bridge axes in partial crosssections of the bridge.
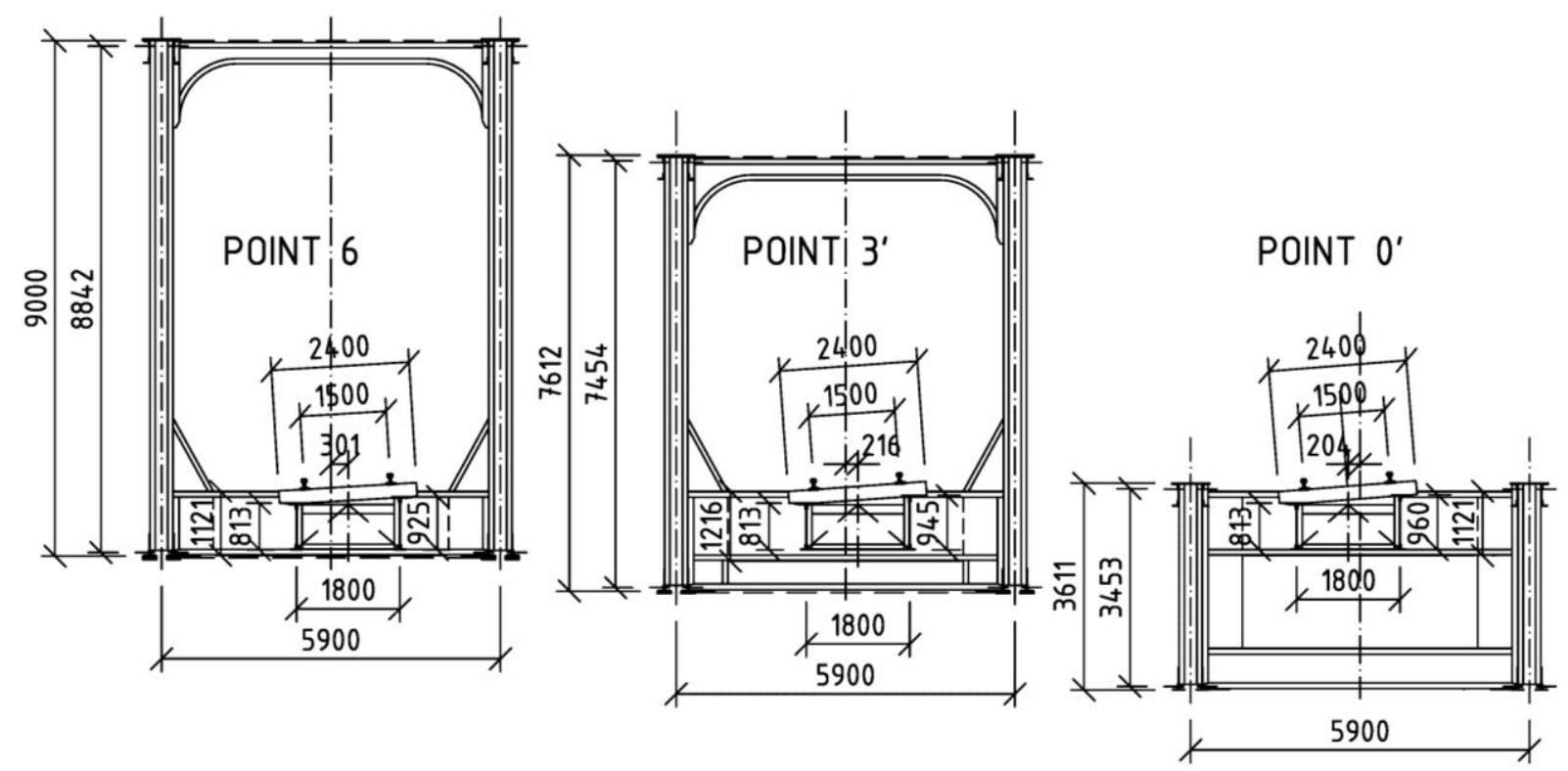

Fig. 3: Transversal disposition of the truss bridge in the medium span.

\section{Observed joints}

The connection of the second stringer to the second cross-beam (denoted as $1^{\prime}$ ' in Fig. 2, Fig. 4 and Fig. 5) was chosen for experimental measurements, as well as the connection of the same 
cross-beam ( $\left.1^{\prime}\right)$ to the main truss girder. Arrangement of the stringer-to-cross beam connection as well as the location of strain gauges at the stringer is obvious from Fig. 4.

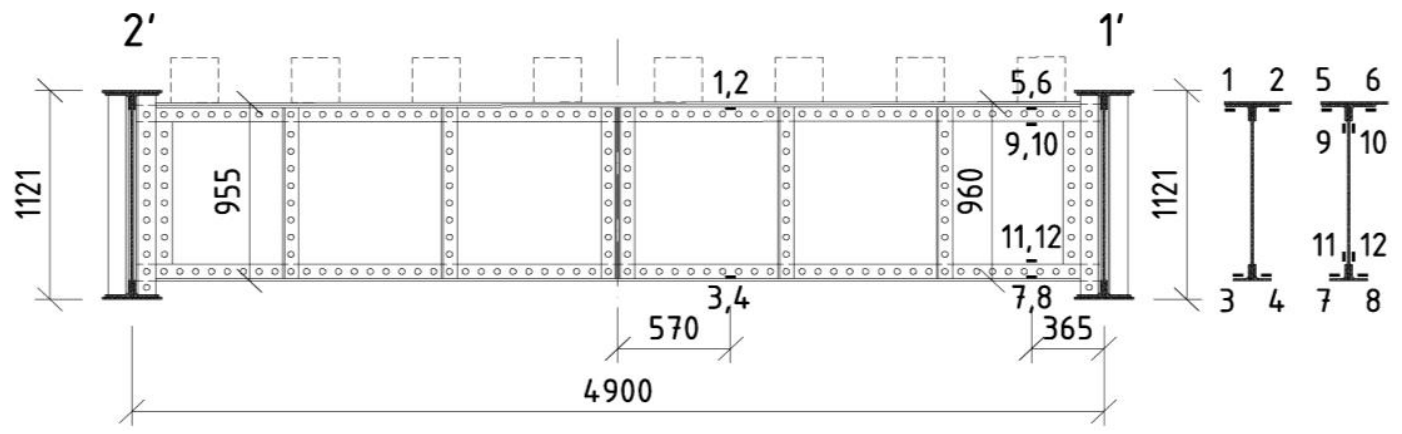

Fig. 4: Arrangement of strain gauges at the stringer.

Arrangement of the bridge cross section at the point 1', including the connection of the crossbeam to the main truss girder, as well as the location of strain gauges at the cross beam are obvious from Fig. 5.

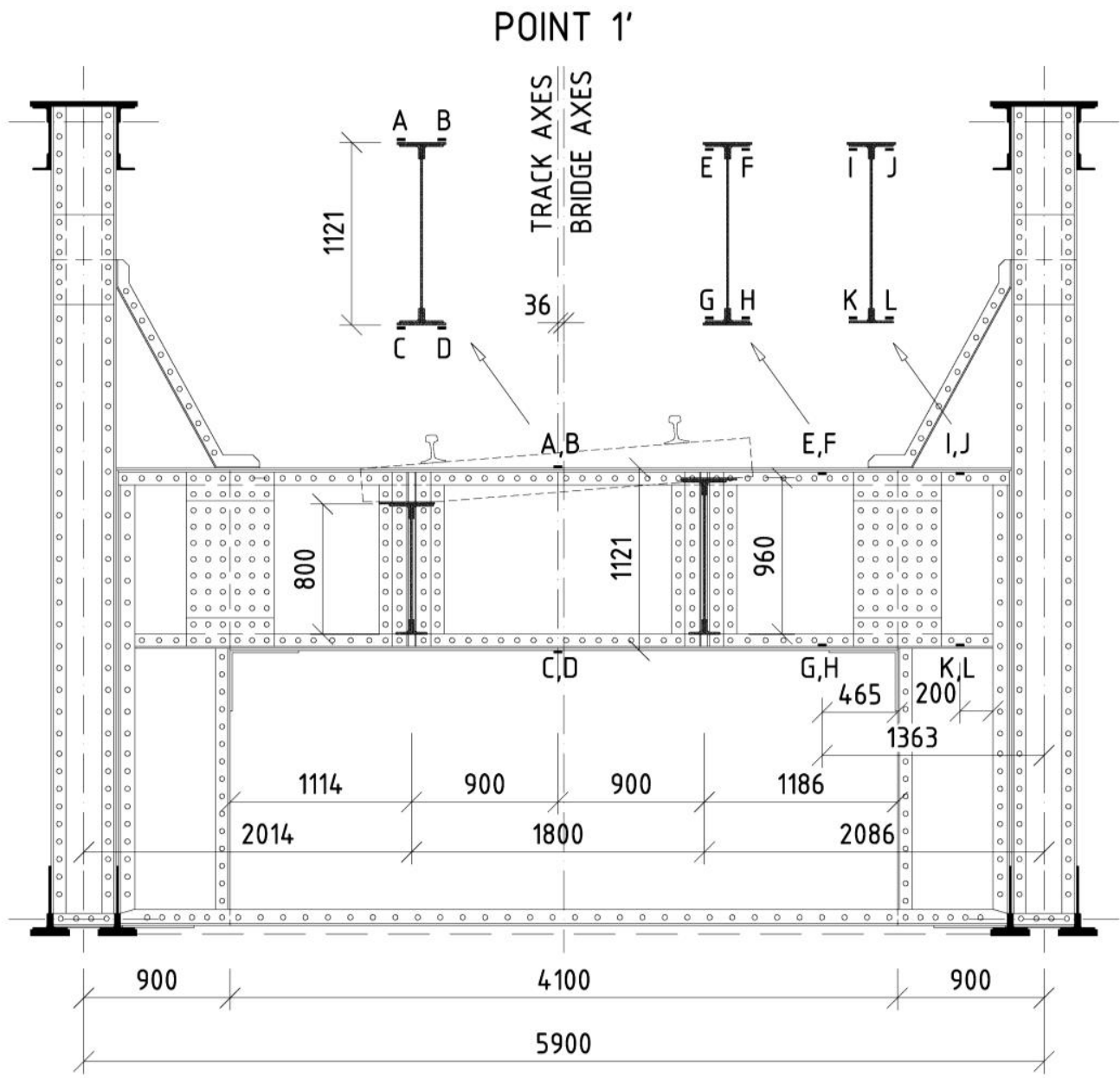

Fig. 5: Bridge cross section at the point 1' and arrangement of strain gauges at the cross-beam. 


\section{Experimental measurements on the bridge}

The experimental measurements on the bridge were realized in October 2014. The aim was to obtain the stress response of the observed bridge deck members to the actual traffic load. Within the accomplished measurements, passages of 11 trains were recorded, from which there were 3 freight trains and 8 passenger trains. The recorded courses of stress response to the traffic loads in the individual measured points were graphically processed using Microsoft Excel software. The example of recorded courses of the stress response caused by passage of a freight train in the observed points in the stringer and cross-beam, respectively, are demonstrated in Fig. 6 and Fig. 7, respectively. The individual observed points are denoted in accordance with Fig. 4 and Fig. 5, respectively, for example SG_01 represents the strain gauge 1, etc.

In Fig. 6, there are not presented the stresses in the upper flanges of stringer near the crossbeam. Unfortunately, the corresponding strain gauges denoted as SG_05 and SG_06 have not given the correct stress values, which was probably caused by damaging the bond between the strain gauge and the steel surface.
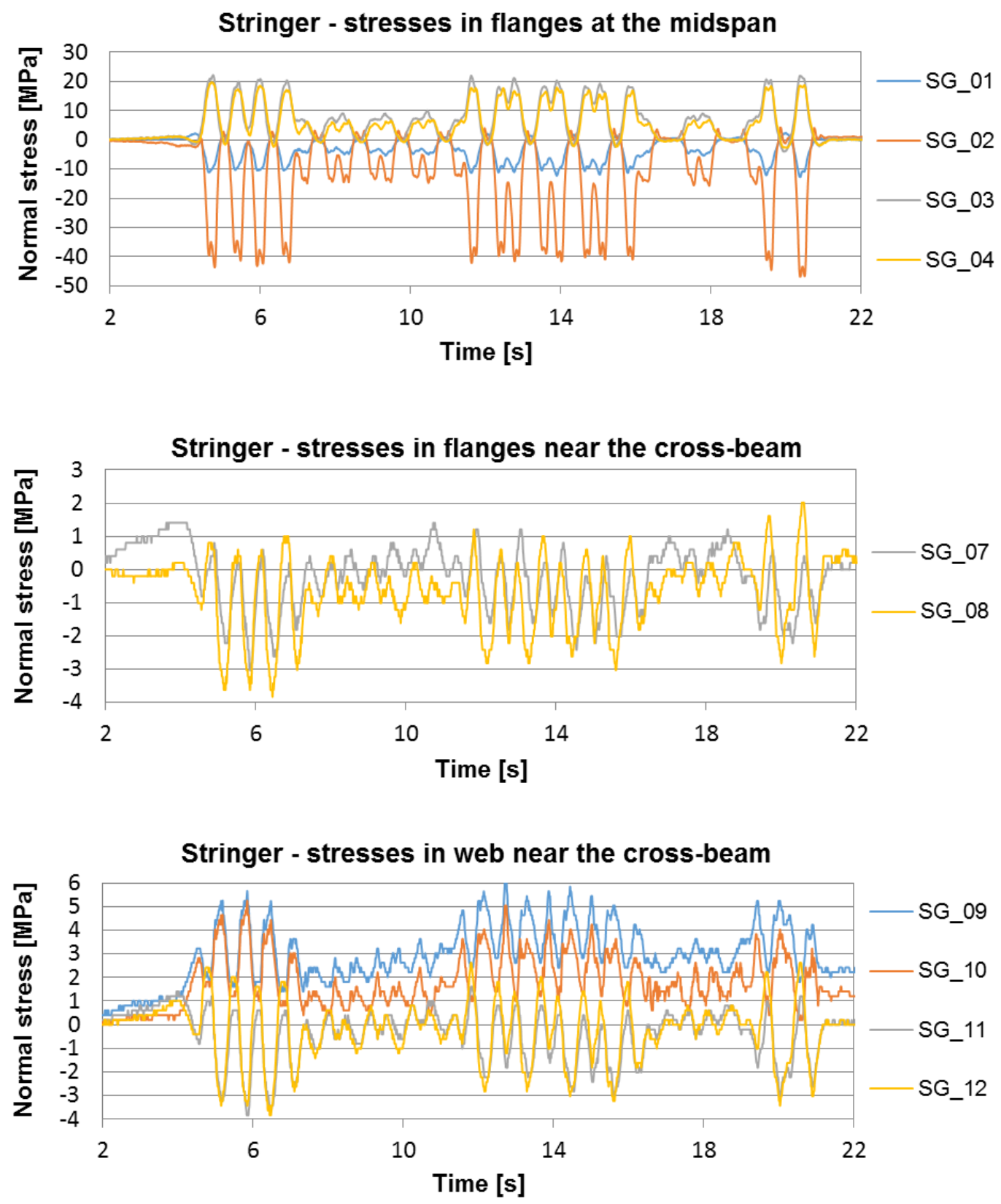

Fig. 6: Measured courses of stress response in the stringer. 
With regard to the arrangement of the stringer-to-cross beam connection (Fig. 4), the hinged behaviour of the joint could be generally assumed on its design. While in the horizontal direction this can be clearly accepted, in the vertical direction there can be expected a certain rigidity. This assumption has been confirmed by the measured values of the longitudinal normal stress in the cross section near the cross-beam. Although the normal stress values in the upper flange have not been recorded correctly, the predominant compressive stress values measured in the lower flange (SG_07 -SG_08) as well as the normal stress values measured in the wall of the stringer in the same cross section (SG_09 - SG_12) indicate occurrence of negative bending moments, which may occur only due to the vertical joint stiffness.
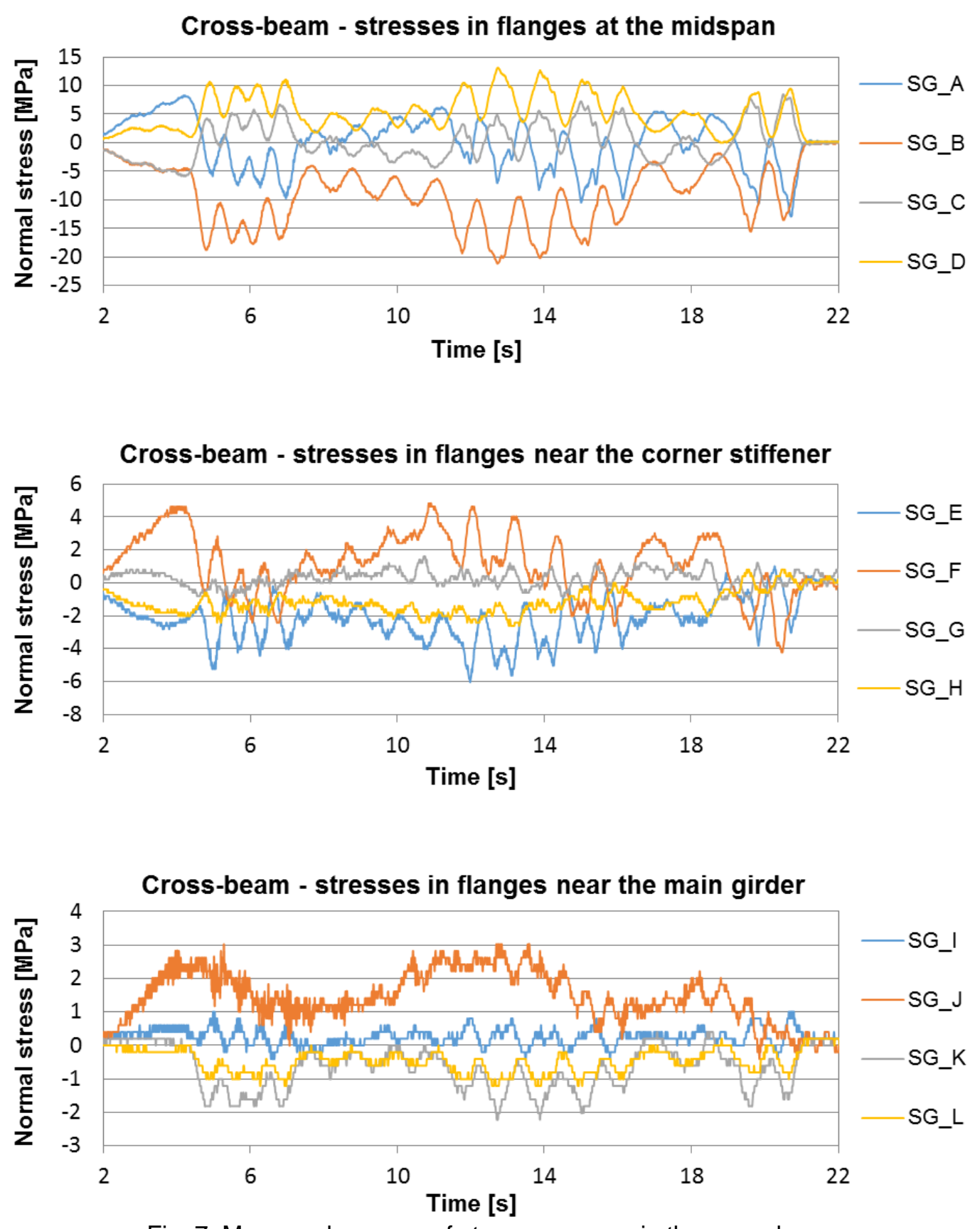

Fig. 7: Measured courses of stress response in the cross-beam.

With regard to the cross-beam-to-main-girder connection arrangement, a similar behaviour of the joint could be expected, however, with signifficantly higher stiffness in vertical direction. This 
assumption has also been confirmed by experimental measurements. It can be demonstrated on the stress values measured in the cross-section located near the corner stiffener, which are presented in the central graph in Fig. 7 (SG_E - SG_H). Considering the fact that the mean stress values in the upper flange as well as in the bottom flange oscillate approximately around zero, it is obvious that this cross section (located $465 \mathrm{~mm}$ from the support) corresponds approximately to a location with zero vertical bending moment on the cross-beam.

The processed results of experimental measurements represent the first step in analysing stiffness of the investigated connections. The preliminary conclusions presented above are only indicative, especially when we consider that the recorded stress response courses are significantly affected by horizontal load effects (centrifugal force, nosing force) as well as by spatial behaviour of the whole superstructure (cooperation of the ridge deck members with the main trusses). The more serious conclusions on the stiffness of both observed connections may be put only after a thorough numerical analysis, which will be the subject of subsequent research.

\section{Conclusions}

In the context of research aimed at the actual stiffness of riveted steel railway bridge deck members' connections, there have been realized experimental measurements on the chosen steel truss bridge. The aim of experimental research was to obtain time courses of stress response of the selected bridge deck members to the traffic load. The results of experimental measurements essentially confirmed the expected behaviour of the investigated joints. The measured stress courses will be used for calibration of a numerical computational model, which will be subsequently used for further theoretical analysis of stiffness of the observed connections.

\section{Acknowledgement}

This work was supported by the Slovak Research and Development Agency under contract APVV-0106-11 and by Research Project No. 1/0364/12 of Slovak Grant Agency. Moreover, the research presented here was supported by the European Regional Development Fund and the Slovak state budget for the project "Research Centre of University of Žilina", ITMS 2622022018.

\section{References}

[1] VIČAN, J. - GOCÁL, J. - JOŠT, J.: Fatigue resistance of typical fatigue prone riveted steel railway bridge structural detail. Communications - Scientific letters of University of Zilina, Vol. 13, No. 3/2011, University of Zilina, EDIS.

[2] VIČAN, J. - JOŠT, J. - GOCÁL, J.: Determination of Fatigue Category and Numerical Analysis of the Stringer to Crossbeam Connection of Riveted Steel Railway Bridges. 23rd Czech and Slovak International Conference „Steel structures and bridges 2012“, Podbanské, Slovakia, 26.28.09.2012. Procedia Engineering, vol. 40/2012.

[3] VIČAN, J. - JOŠT, J. - GOCÁL, J.: Analysis of the stringer-to-cross-beam riveted joints behaviour. Civil and Environmental Engineering, Vol. 10, Issue 1/2014, 50-60 DOI: 10.2478/cee-2014-0007.

[4] ADAMSON, D. - KULAK, G.: Fatigue tests of riveted bridge girders. University of Alberta. Structural engineering report no. 210, August, 1995.

[5] MATAR, E. : Evaluation of fatigue category of riveted steel bridge connections. Structural Engineering International 1/2007, Science and technology, pp. 72-78.

[6] TARAS, A. - GREINER, R.: Development and application of a fatigue class catalogue for rivited bridge components. Structural Engineering International 1/2010, Science and technology, pp. 91103. 